\title{
Placebos mit Heilkraft? Placeboeffekt und -einsatz in der (Schmerz-)Therapie
}

Norbert Roewer, Peter Kranke

\author{
„Doch als er mich fragte, ob ich das Mittel wider das Kopfweh wisse, antwortete ich \\ nicht ohne Mühe, ich wisse es. Worin besteht es nun? fuhr Charmides fort. Und ich \\ erwiderte, dass es ein Blatt sei, zu dem Mittel aber noch ein Spruch gehöre, und dass, \\ wenn jemand diesen spreche, beim Gebrauch von jenem das Mittel durchaus gesund \\ mache; ohne den Spruch aber sei das Blatt nichts nütze.“ \\ Platon, Charmides 155C/156A, übersetzt von L. Georgii (gefunden in [1])
}

Was Plato mit seinen Ausführungen in „Charmides“ kundtat, beschäftigte und beschäftigt Ärzte, Therapeuten und Forscher seit Jahrhunderten und es scheint kein Ende in Sicht. Faszinierend und befremdlich zugleich erscheint nicht wenigen Kollegen der tagtäglich und beinahe inflationär verwendete Begriff des „Placebos“ (Placebo: lat. „ich werde gefallen“) oder des „Placeboeffekts“. Meist schwingt eine negative Konnotation mit, wenn über das Präfix „Placebo-“ eine Wirkung relativiert oder gar abgewertet wird. Das Internet „kennt“ den Placebobegriff zur Genüge, wenn in der Verbindung „Placebo AND medicine“ über 30 Millionen Treffer auftauchen. Nicht zuletzt hat nahezu jeder von uns eine Vorstellung davon, was Placebo und Placeboeffekt ist. Bei genauem Hinsehen und Nachfragen verliert das Bild gleichwohl an Schärfe und nicht selten macht sich Unsicherheit breit, wenn es um die Definition im Konkreten geht.

\section{Was versteht man unter Placeboeffekt?}

Vielfach ist die erste Assoziation jene zu einer klinischen Studie, in der ein Prüfpräparat oder mehrere (aktive) Interventionen gegen eine (scheinbar) unwirksame Kontrolle, nämlich das Placebo, getestet werden, um schlussendlich den durch das „Verum“ induzierten pharmakologischen Effekt zu determinieren ( $\triangleright$ Abb. 1). Viel weniger präsent dem gegenüber dürfte der allägliche Placeboeffekt in der Arzt-Patienten-Beziehung im Bewusstsein verankert sein, der nicht durch eine Droge im eigentlichen Sinne, sondern eben durch die sog. „Droge Arzt“ induziert wird. Ein Effekt, der durchaus erwünscht ist und dessen Auswirkungen noch dazu nicht in Hinblick auf Risiken und Nebenwirkungen aufklärungspflichtig sind.
Oder vielleicht doch? Und wie verhält es sich mit dem Noceboeffekt? Hiermit ist der Effekt gemeint, der sich dann einstellt, wenn mit einer therapeutischen Maßnahme eine negative körperliche oder kognitive Reaktion assoziiert wird. Wird beim Placeboeffekt durch eine positive Erwartungshaltung infolge einer Maßnahme wie z. B. Medikamentengabe oder operative Behandlung ein günstiger Einfluss auf den Krankheitsverlauf eines Patienten genommen, so wird beim Noceboeffekt möglicherweise ein Schaden induziert ohne ein Agens, dem wir gemeinhin eine schädigende Wirkung zubilligen.

Die Begriffsverwirrung fängt bereits bei der Zuschreibung der Effekte zum vermeintlichen Placeboeffekt an. Denn vieles, was mitunter auch in klinischen Studien dem Placeboeffekt zugeschrieben wird, fällt im strengen wissenschaftlichen Sprachgebrauch gar nicht darunter und ist eher statistischen Effekten ${ }^{1}$ oder dem natürlichen Krankheitsverlauf zuzurechnen ( $\triangleright$ Abb. 2).

Vom eigentlichen Placeboeffekt ist die Placeboreaktion abzugrenzen. Der Placeboeffekt im eigentlichen Sinne ist der Varianzanteil in der Placeboreaktion, der ausschließlich auf die Placebogabe zurückzuführen ist und der notwendigerweise kleiner als die Gesamtreaktion ausfällt, weil er sich aus der Subtraktion aller potenziellen konfundierenden Faktoren vom Gesamteffekt errechnet. Die Placeboreaktion wird landläufig gerne als Placeboeffekt bezeichnet, spiegelt in strenger Lesart aber die Gesamtheit der gemessenen Veränderung nach Gabe von Placebo wider. Sie umfasst folglich ein breites Spektrum von

Stichwort „Regression zum Mittelwert“ (engl. regression to the mean): statistisches Phänomen, bei dem die Zweitmessungen weniger extrem ausfallen als Erstmessungen. Zweitmessungen haben also die Tendenz zum Mittelwert, d.h. sie fallen kleiner aus. 


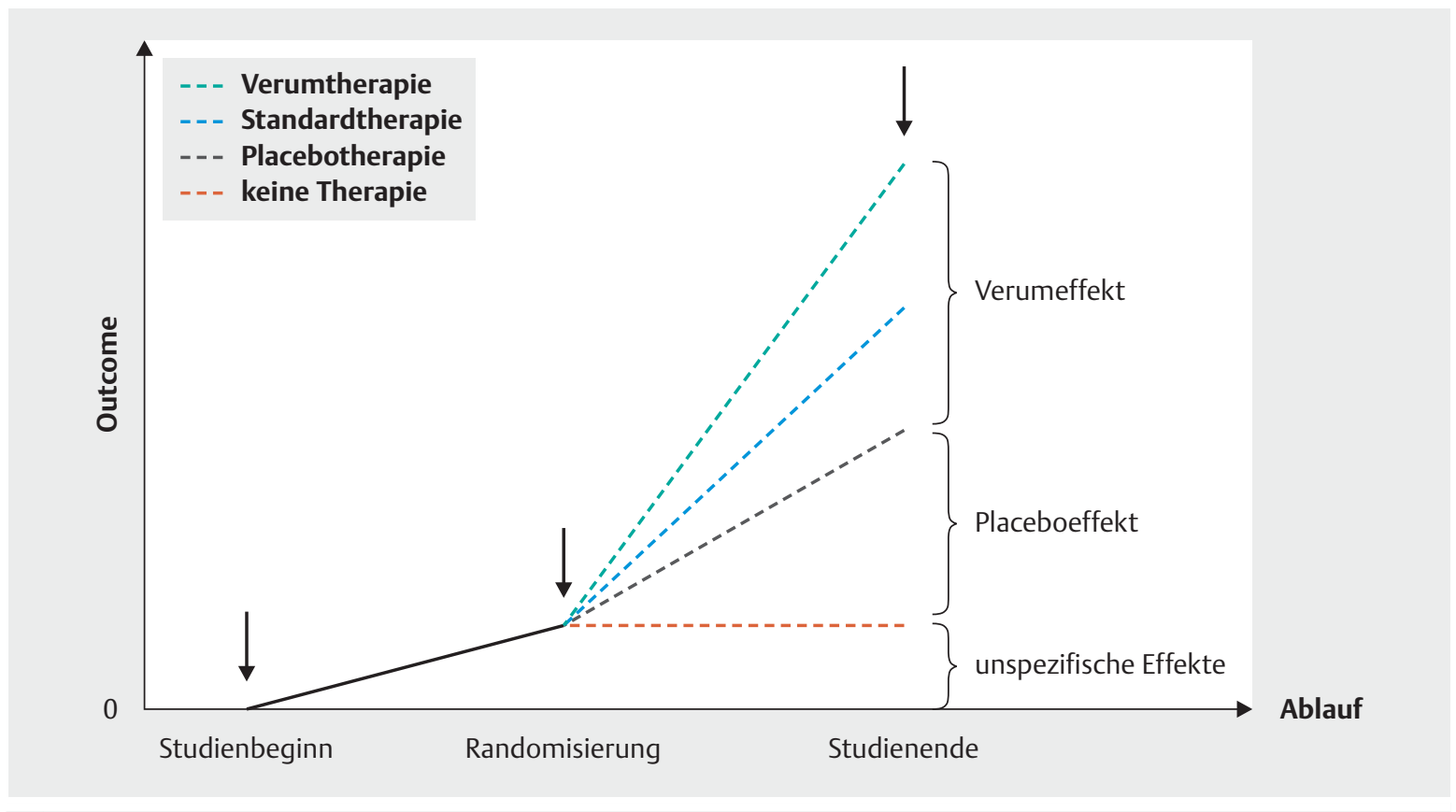

- Abb. 1 Möglicher Verlauf von Studieneffekten unter Berücksichtigung der Anteile „Placeboeffekt“”, „Verumeffekt“ und weiteren „unspezifischen Effekten“ [2].

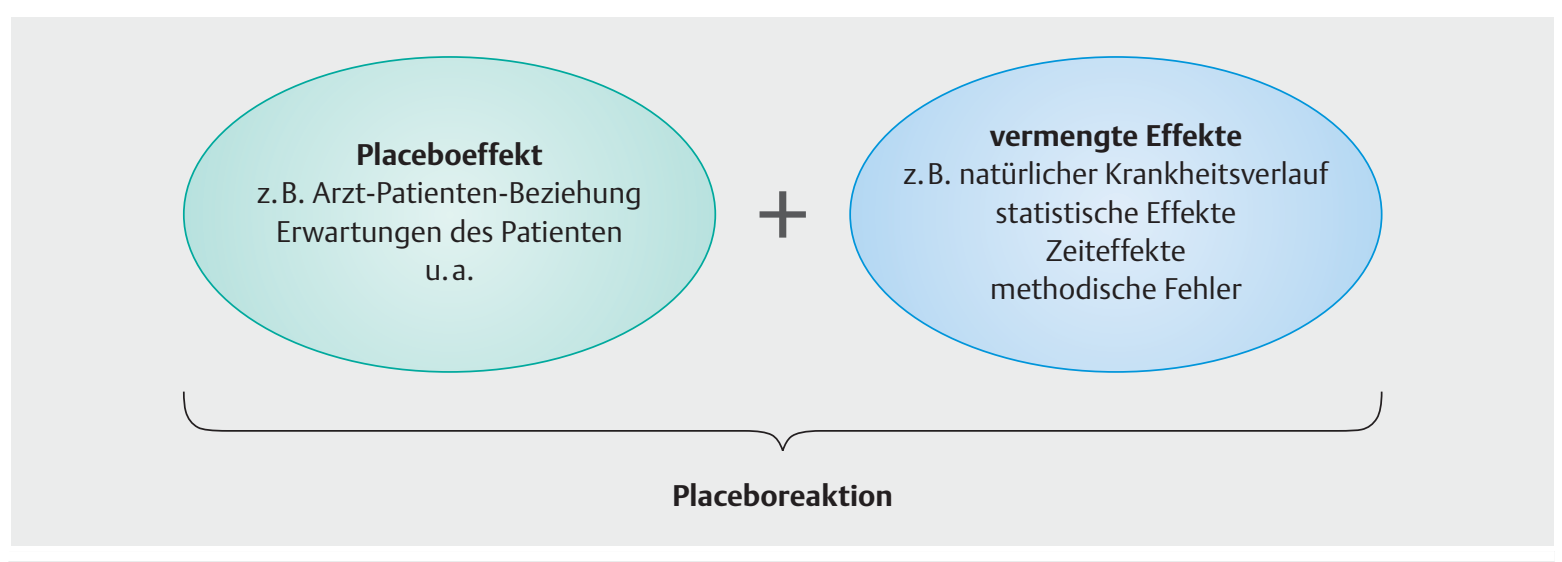

Abb. 2 Beziehung zwischen Placeboeffekt und Placeboreaktion [3].

Effekten, die nicht alle interventionsspezifisch sind und daher als vermengte Faktoren ( $\bullet$ Abb. 2, Tab. 1) gesehen werden müssen.

Das neu erwachte Interesse an der Thematik an sich [58], der vermehrte klinische Einsatz von Placebo und der mutmaßlich im klinischen Alltag nicht selten zu beobachtende Noceboeffekt (im stets weiter ausufernden Impetus, vermeintlich alle und jede erdenklichen Effekte aufklären zu müssen) waren Grund genug, dem Thema „Placebo“ ein eigenes Topthema zu widmen. Und zwar exemplarisch an der Säule unseres Faches (Schmerztherapie), die eine enge Verbindung zum Thema Placebo und Place- boforschung aufweist, seit Lasagna und Beecher in frühen Arbeiten auf den Placeboanteil von Analgetika hingewiesen haben $[9,10]$. So finden sich in der vor Ihnen liegenden AINS-Ausgabe Ausführungen zu einer ganzen Reihe von Aspekten, die im Kontext von Placebo, aber auch dem Placeboeinsatz und der Placeboforschung interessant und wissenswert erscheinen.

\section{Placebo in der Akutschmerztherapie}

Im 1. Beitrag von Roland Haaga und Alexander Schnabel werden die Placebo- und Noceboeffekte in der Akutschmerztherapie in den Mittelpunkt gestellt [11]. Aus- 
- Tab. 1 Überblick über die Begrifflichkeiten im Kontext von Placebo und Placebowirkungen [4].

\begin{tabular}{|l|l|}
\hline $\begin{array}{l}\text { Bezeich- } \\
\text { nung }\end{array}$ & $\begin{array}{l}\text { Wirkung bzw. Wirksamkeit der } \\
\text { Intervention }\end{array}$ \\
\hline Placebo & pharmakologisch unwirksame Substanz \\
\hline $\begin{array}{l}\text { Pseudo- } \\
\text { placebo }\end{array}$ & $\begin{array}{l}\text { pharmakologisch aktive Substanz, die } \\
\text { aber keine spezifischen Effekte (fehlende } \\
\text { Evidenz) für die betreffende Indikation hat }\end{array}$ \\
\hline $\begin{array}{l}\text { Placebo- } \\
\text { effekt }\end{array}$ & $\begin{array}{l}\text { Effekt von u. a. Erwartung, Erfahrung, } \\
\text { Arzt-Patienten-Beziehung }\end{array}$ \\
\hline $\begin{array}{l}\text { Placebo- } \\
\text { reaktion }\end{array}$ & $\begin{array}{l}\text { Placeboeffekt + andere Effekte (z. B. na- } \\
\text { türlicher Krankheitsverlauf, statistische Ef- } \\
\text { fekte, Zeiteffekte, methodische Fehler) }\end{array}$ \\
\hline
\end{tabular}

gehend von den involvierten Neuro- sowie psychophysiologischen Prozessen werden die beteiligten Signaltransduktionswege dargestellt und es wird auf die 3 essenziellen psychologischen Mechanismen im Zustandekommen des Placebo- und Noceboeffekts hingewiesen:

1. die klassische Konditionierung

2. das soziale Lernen

3. die Schmerzerwartung

Die Autoren schlussfolgern, dass eine offene Analgetikagabe, eine ausführliche Aufklärung über Wirkung und Nebenwirkung - gleichwohl ohne Letztere zu sehr in den Fokus zu stellen - im perioperativen Bereich bei der Medikamentengabe (Analgetika), aber auch bei der Aufklärung in Hinblick auf andere Interventionen am ehesten zu einer additiven Verbesserung der Akutschmerztherapie führt.

\section{Placebo-Nocebo-Ethik in der Patientenkommunikation}

Nachfolgend erörtern die Autoren um Daniel R. Friedrich im Beitrag „Placebo-Nocebo-Ethik in der Patientenkommunikation“ die Placebo- und Noceboeffekte im Spannungsfeld medizinethischer Prinzipien [12]. Sie heben nochmals hervor, dass die Wirksamkeit von Interventionen zumindest teilweise durch kontextuelle Veränderungen der konkreten Behandlung beeinflusst werden kann. Daraus folgt, dass die Festlegung einer „objektiven“ Wirkung von Interventionen nahezu unmöglich ist. Ferner liegt ein Augenmerk auf dem Zielkonflikt zwischen Autonomieförderung und leidensminderndem Erwartungsmanagement. Ein wesentliches Fazit der Autoren ist, dass auch im Rahmen der anästhesiologischen Aufklärung bei zu erwartendem „Nettonutzen“ einer Intervention deren positive Wirkungen auch inhaltlich und sprachlich in den Vordergrund gestellt werden sollte - ein Umstand, der gerade vor dem Hintergrund der vielfach auf juristische Akkuratesse getrimmten Aufklärung vielen von uns nicht gerade leichtfallen dürfte. Interessant und überzeugend in Hinblick auf die Befürwortung einer positiven Diktion im Aufklärungsgespräch ist in diesem Kontext die Gegenüberstellung mit den vielzitierten „Beipackzetteln“ von Medikamenten, die sich in der Regel mit über $90 \%$ ihres Textes den Nebenwirkungen von Arzneimitteln widmen, während weniger als $10 \%$ auf die positiven Aspekte der Pharmaka hinweisen.

\section{Placeboeffekte bei chronischen Schmerzen: Forschungsstand und klinische Anwendung}

Abschließend führen Maike Müller und Anne-Kathrin Bräscher [13] aus, wie Placeboeffekte auch in der Therapie chronischer Schmerzen hineinspielen und unter bestimmten Umständen auch gezielt genutzt werden können. Im Anschluss an eine Einführung des Begriffs der „Placebohypoalgesie“ als unspezifisches „Additivum“, das den spezifischen pharmakologischen Effekt eines Analgetikums bedeutsam steigern kann, werden zunächst die experimentellen Placebodesigns in klinischen Studien erläutert, auf die im Folgenden immer wieder Bezug genommen wird. Die zugrunde liegenden Mechanismen und neurobiologischen Grundlagen der Placebohypoalgesie kommen in dem praxisorientierten Beitrag ebenso zur Sprache wie gezielte Tipps, wie jene Placebohypoalgesie konkret im klinischen Alltag verstärkt werden kann. Einmal mehr wird anhand der zahlreichen referenzierten Belege deutlich, dass „Pharmakotherapie“ oder „Therapie“ im Allgemeinen eben mehr ist als das Verschreiben bunter Pillen seitens des Therapeuten bzw. der Konsum derselben durch den Patienten, sondern vielmehr ein ausgesprochen komplexes Interaktionsmuster darstellt.

\section{Placeboeinsatz in der Medizin}

Zurück zu Placebo im Allgemeinen: Die am häufigsten zitierte Definition zu Placebo dürfte nach wie vor von Shapiro aus dem Jahr 1964 stammen [14]. Als Placebo in dieser Definition ist jegliche therapeutische Prozedur definiert, die durchgeführt wurde, um einen Effekt zu erreichen, der für die behandelte Erkrankung gleichwohl objektiv ohne spezifische Aktivität auf die Symptome, die Erkrankung oder das Syndrom des Patienten ist.

Diese Definition erfolgte unter der Annahme, dass Placebos inert sind und mithin keinerlei spezifische Effekte haben. Und genau jene Annahme ist heutzutage nicht mehr haltbar. Da von spezifischen Placeboeffekten ausgegangen werden muss, hat nicht zuletzt die Arbeitsgruppe des Wissenschaftlichen Beirats der Bundesärztekammer entschieden, in einer umfassenden Abhandlung zur Thematik für die klinische Forschung und die tägliche Patien- 
tenbetreuung demgegenüber einen eher weitgefassten Placebobegriff zu verwenden: „Dieser bezieht sich nicht nur auf die Gabe eines Scheinmedikaments bzw. den Einsatz einer Scheinprozedur. Auch bei einer Behandlung mit Verum ist ein Placeboeffekt vorhanden, der durch das jeweilige Behandlungsumfeld, die Erwartungen des Patienten und des Arztes sowie die unterschiedlich gelungene Arzt-Patienten-Interaktion moduliert wird.“ [3]

In dieser umfassenden wie aufschlussreichen Stellungnahme wird u. a. auf eine Differenzierung abgestellt:

- zwischen den als „echten“ oder „reinen“ Placebos als „Scheinmedikamente, die nur eine pharmakologisch unwirksame Substanz und ggf. auch Hilfsstoffe wie Geschmackskorrigenzien oder Farbstoffe enthalten“, und

- den sog. „Pseudoplacebos“ ( $\triangleright$ Tab. 1), die auch als „unreine Placebos“ bezeichnet werden. Hierbei handelt es sich um „pharmakodynamisch aktive Substanzen, die allerdings bei der Erkrankung keine spezifische Wirksamkeit entfalten, entweder weil die Dosis zu niedrig ist oder die behandelte Erkrankung nach herrschender Lehrmeinung nicht darauf anspricht.“ [3]

Gerade letztere „Placebovariante“ dürfte in der täglichen Praxis von noch größerer Relevanz sein, da reine Placebos aufgrund rechtlicher Vorgaben nur im Ausnahmefall eingesetzt werden können.

Aber auch hier stellt sich die Frage nach der Zulässigkeit. Denn es „besteht grundsätzlich die Pflicht, eine wirksame Therapie anzuwenden und dadurch gesundheitlichen Schaden vom Patienten abzuwenden oder Schaden zu begrenzen“. In der zur Lektüre empfohlenen Stellungnahme der Bundesärztekammer sowie der dazu erschienenen Veröffentlichung im Deutschen Ärzteblatt wird gleichwohl darauf verwiesen, dass bei Aufklärung grundsätzlich auch eine Placebomedikation infrage kommt: „Stehen mehrere gleich geeignete Vorgehensweisen mit unterschiedlichem Risiko zur Verfügung, ist diejenige zu wählen, welche den Heilerfolg am besten gewährleistet und in den damit verbundenen Nebenwirkungen am wenigsten schädigend ist. Dies gilt auch für den Einsatz von Placebo.“ [4]

Interessant sind diese Ausführungen auch insofern, als gemäß den nachzulesenden Ausführungen „nicht wenige Therapeuten - in diesem Fall Ärzte aus unterschiedlichen Abteilungen einer Klinik der Maximalversorgung - zumindest gelegentlich, teilweise auch häufig Placebos einsetzen. Schmerzen wurden am häufigsten als Grund für eine Placebogabe angeben, danach folgen: Schlaflosigkeit, depressive Verstimmung, Verdauungsstörungen“ [1].
Weiter wird ausgeführt, dass unter Schweizer Hausärzten der Placeboeinsatz nicht unüblich ist und, wenn er erfolgt, dann größtenteils auf Pseudoplacebos (57\%) zurückgegriffen wird.

Die Anwendung von Placebo in der klinischen Forschung ist generell zulässig und oft auch im Rahmen von Zulassungsverfahren gefordert. Voraussetzung für den Einsatz, z. B. in Arzneimittelstudien, ist, dass der Patient rechtswirksam in die Möglichkeit der Teilnahme an einem Placeboeinsatz eingewilligt hat, wodurch auch die Strafbarkeit entfällt.

\section{Noch offene Fragen}

Gleichwohl ist auch in der klinischen Forschung noch lange nicht geklärt, welche Anteile einer Placeboreaktion durch welchen Aspekt der therapeutischen Interaktion und des eigentlichen Placeboeffekts bedingt werden. Nicht umsonst eröffnete der Editor-in-Chief des Journals Clinical Therapeutics jüngst, dass mit Beginn des Jahres 2018 die Placebobeschreibung alleine nicht ausreicht. Vielmehr sollen im Rahmen eines konsolidierten Berichts und im Sinne des transparenten Studienreportings auch Farbe, Typ (Kapsel, Pille, Flüssigkeit), Form (bei festen Placebos), Inhalt (z. B. Milchzucker), Geschmack und Verpackung (z.B. Double-Dummy-Technik) vermerkt und mitgeteilt werden [15].

Ob diese Maßnahmen schlussendlich zur Erhellung des therapeutischen Effekts beitragen, ist offen. Möglicherweise stellen in der Retrospektive und bei Betrachtung mehrere Studien dann diese Zusatzinformationen essenzielle Puzzleteile in der Aufklärung dessen dar, was Platon skizzierte und Beecher mit dem Begriff des „Powerful Placebo" umschrieb.

Sie sehen, es sind bei weitem noch nicht alle Fragen zur Placebowirkung und zum Placeboeinsatz geklärt. Dennoch wünschen wir Ihnen einstweilen eine interessante und erkenntnisreiche Lektüre der Beiträge des Topthemas, nicht zuletzt mit dem Ziel, den (hilfreichen) Placeboeffekt in der Alltagsarbeit nutzbringend einzusetzen. Bei weiterführendem Interesse sei nochmals auf die umfassende Lektüre „Placebo in der Medizin“ verwiesen [1], herausgegeben von der Bundesärztekammer auf Empfehlung ihres Wissenschaftlichen Beirates. Diese kann käuflich erworben werden, steht aber zudem auf der Homepage der Bundesärztekammer zum Download zur Verfügung: http://www.bundesaerztekammer.de/ fileadmin/user_upload/downloads/Placebo_LF_1_ 17012011.pdf

Herzlich Ihre

Norbert Roewer und Peter Kranke 


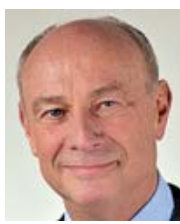

\section{Norbert Roewer}

Univ.-Prof. Dr. Dr. h. c., Direktor und Ordinarius an der Klinik und Poliklinik für Anästhesiologie des Universitätsklinikums Würzburg und stellvertretender ärztlicher Direktor des Universitätsklinikums Würzburg. Er ist Mitglied im engeren Präsidium der DGAI. Prof. Roewer gehört zum Herausgeberteam der AINS.

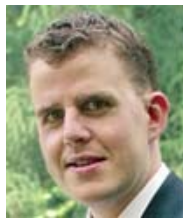

\section{Peter Kranke}

Univ.-Prof. Dr. med., MBA, Oberarzt, Leiter der klinischen Forschung und Lehrbeauftragter an der Klinik und Poliklinik für Anästhesiologie des Universitätsklinikums Würzburg. Sein klinischer Verantwortungsbereich ist die Anästhesie in der Gynäkologie und Geburtshilfe.

Korrespondenzadresse

Univ.-Prof. Dr. Peter Kranke, MBA

Klinik und Poliklinik für Anästhesiologie

Universitätsklinikum Würzburg

Oberdürrbacher Straße 6

97080 Würzburg

kranke_p@ukw.de

\section{Literatur}

[1] Bundesärztekammer auf Empfehlung ihres Wissenschaftlichen Beirats. Placebo in der Medizin. Köln: Deutscher Ärzteverlag; 2011

[2] Bundesärztekammer auf Empfehlung ihres Wissenschaftlichen Beirats. Bedeutung des Placebos für die Evidenz-basierte Medizin. In: Placebo in der Medizin. Köln: Deutscher Ärzteverlag; 2011: 68-78

[3] Bundesärztekammer auf Empfehlung ihres Wissenschaftlichen Beirats. Definitionen, Begrifflichkeiten, Abgrenzungen. In: Placebo in der Medizin. Köln: Deutscher Ärzteverlag; 2011: 3-17
[4] Stellungnahme des Wissenschaftlichen Beirats der Bundesärztekammer: „Placebo in der Medizin“. Dtsch Arztebl 2010; 107: A 1417/B-1253/C-1233

[5] Dodd S. Current insights in the placebo and nocebo phenomena. Clin Ther 2017; 39: 456-457

[6] Dodd S, Dean OM, Vian J et al. A review of the theoretical and biological understanding of the nocebo and placebo phenomena. Clin Ther 2017; 39: 469-476

[7] Chavarria V, Vian J, Pereira C et al. The placebo and nocebo phenomena: their clinical management and impact on treatment outcomes. Clin Ther 2017; 39: 477-486

[8] Enck P, Klosterhalfen S, Weimer K. Unsolved, forgotten, and ignored features of the placebo response in medicine. Clin Ther 2017; 39: 458-468

[9] Lasagna L, Mosteller F, Von Felsinger JM et al. A study of the placebo response. Am J Med 1954; 16: 770-779

[10] Beecher HK, Keats AS, Mosteller F et al. The effectiveness of oral analgesics (morphine, codeine, acetylsalicylic acid) and the problem of placebo "reactors" and "non-reactors". J Pharmacol Exp Ther 1953; 109: 393-400

[11] Haaga R, Schnabel A. Placebo in der Akutschmerztherapie. Anästhesiol Intensivmed Notfallmed Schmerzther 2018; 53: 579-590

[12] Friedrich DR, Reichardt J-O, Rittner H. Placebo-Nocebo-Ethik in der Patientenkommunikation. Anästhesiol Intensivmed Notfallmed Schmerzther 2018; 53: 592-604

[13] Müller M, Bräscher A-K. Placeboeffekte bei chronischen Schmerzen: Forschungsstand und klinische Anwendung. Anästhesiol Intensivmed Notfallmed Schmerzther 2018; 53: 605-617

[14] Shapiro AK. Etiological factors in placebo effect. JAMA 1964; 187: $712-714$

[15] Shader RI. Placebos and clinical therapeutics. Clin Ther 2017; 39: 1274

Bibliografie

DOI https://doi.org/10.1055/a-0636-1776

Anästhesiol Intensivmed Notfallmed Schmerzther 2018; 53: 574-578 @ Georg Thieme Verlag KG Stuttgart · New York ISSN 0939-2661 